\title{
U na estrategia simple para mejorar la búsqueda de artículos indexados en SciELO
}

\author{
A simple strategy to improve \\ searching of indexed articles \\ in SCiELO
}

\begin{abstract}
Sr. Editor: SciELO (Scientific Electronic Library Online) es una biblioteca electrónica que Scubre una colección seleccionada de revistas científicas y en la cual participan ocho países de Ibero América (Argentina, Brasil, Chile, Colombia, Cuba, España, Portugal y Venezuela; además de SciELO Salud Pública y SciELO Ciencias Sociales); existiendo iniciativas en desarrollo en otros cinco países (Costa Rica, México, Paraguay, Perú y Uruguay; además de Ciencia y Cultura, Innovación UNIEMP, Pesquisa FAPESP y West Indian Medical Journal) ${ }^{1}$. La Revista Médica de Chile fue la primera revista que se incorporó a la colección de SciELO Chile².

La biblioteca SciELO permite el acceso gratuito a información científica y tecnológica, y representa una fuente importante de consulta complementaria a bases de datos como, por ejemplo, LILACS (Literatura Latinoamericana y del Caribe en Ciencias de la Salud) y MEDLINE (Medical Literature Analysis and Retrieval System Online). Sin embargo, el motor de búsqueda actualmente disponible en el portal SciELO (www.scielo.org) es muy limitado y no permite realizar búsquedas en todas las colecciones de revistas SciELO en un solo paso. Por ejemplo, una dificultad que encontramos es que cuando se realiza la búsqueda en SciELO regional no se incluyen las 57 revistas de los portales de SciELO Cuba y Venezuela, ni las 61 revistas de las iniciativas en desarrollo.

Proponemos una estrategia sencilla y práctica para mejorar las búsquedas en la colección de revistas integrantes de SciELO, utilizando términos booleanos en el buscador Google. Esta estrategia permite buscar, en un solo paso, en las iniciativas SciELO de los siguientes países: Argentina, Brazil, Chile, Colombia, Costa Rica, Cuba, España, México, Paraguay, Perú, Portugal, Uruguay y Venezuela; además de SciELO Salud Pública, SciELO Ciencias Sociales y West Indian Medical Journal (Tabla 1).
\end{abstract}

Correspondencia a: Dr. Walter H. Curioso. Universidad Peruana Cayetano Heredia, Av. Honorio Delgado 430, Urb. Ingeniería. Lima. E mail: wcurioso@u.washington.edu 


\section{Tabla 1. Estrategia de búsqueda en G oogle de artículos en la colección SciELO}

[INSERTAR AQUI LAS PALABRAS O FRASES SEGUN EL TEMA O AUTOR DE INTERES] site:http://www.scielo.cl OR site:http://www.scielo.org.pe OR site:http:// www.scielo.org.ar OR site:http://www.scielo.br OR site:http://www.scielo.org.co OR site:http://scielo.sld.cu OR site:http://scielo.isciii.es OR site:http:// www.scielo.oces.mctes.pt OR site:http://www.scielo.org.ve OR site:http:// www.scielo.org.mx OR site:http://www.scielo.sa.cr OR site:http://scielo.iics.una.py OR site:http://caribbean.scielo.org OR site:http://www.scielo.org.pe OR site:http:// www.scielo.edu.uy OR site:http://www.scielosp.org OR site:http:// socialsciences.scielo.org

Ejemplo 1: Si deseamos buscar artículos en la colección de SciELO relacionados al tema de dispepsia funcional, la estrategia de búsqueda en Google sería:

"dispepsia funcional" site:http://www.scielo.cl OR site:http://www.scielo.org.pe OR site:http://www.scielo.org.ar OR site:http://www.scielo.br OR site:http://www.scielo.org.co OR site:http://scielo.sld.cu OR site:http://scielo.isciii.es OR site:http://www.scielo.oces.mctes.pt OR site:http://www.scielo.org.ve OR site:http://www.scielo.org.mx OR site:http://www.scielo.sa.cr OR site:http://scielo.iics.una.py OR site:http://caribbean.scielo.org OR site:http://www.scielo.org.pe OR site:http://www.scielo.edu.uy OR site:http://www.scielosp.org OR site:http://socialsciences.scielo.org

Ejemplo 2: Si deseamos buscar artículos en la colección de SciELO del Dr. Eduardo Gotuzzo, la estrategia de búsqueda en Google sería:

"Eduardo Gotuzzo" site:http://www.scielo.cl OR site:http://www.scielo.org.pe OR site:http://www.scielo.org.ar OR site:http://www.scielo.br OR site:http://www.scielo.org.co OR site:http://scielo.sld.cu OR site:http://scielo.isciii.es OR site:http://www.scielo.oces.mctes.pt OR site:http://www.scielo.org.ve OR site:http://www.scielo.org.mx OR site:http://www.scielo.sa.cr OR site:http://scielo.iics.una.py OR site:http://caribbean.scielo.org OR site:http://www.scielo.org.pe OR site:http://www.scielo.edu.uy OR site:http://www.scielosp.org OR site:http://socialsciences.scielo.org

Esta estrategia puede ser fácilmente modificable, ya sea añadiendo páginas Webs de futuras iniciativas SciELO, o combinando otras palabras claves o estrategias de búsqueda.

Debido a la rápida expansión del conocimiento médico y de las fuentes de información -en especial la Internet-, los estudiantes de medicina, médicos y otros profesionales de salud con frecuencia presentan dificultades para encontrar información médica de calidad y accesible de manera rápida ${ }^{3,4}$. El personal de salud necesita desarrollar habilidades y conocer herramientas para buscar, recuperar e interpretar críticamente la información para aplicar adecuadamente la medicina basada en evidencias ${ }^{5}$. Hasta que SciELO mejore sus motores de búsqueda, la estrategia propuesta en este trabajo se convierte en una alternativa útil para mejorar las búsquedas bibliográficas en la Internet. 
Agradecimiento

Este trabajo fue realizado en parte con apoyo de un grant del Fogarty/NIH (R01TW007896).

\section{Walter H. Curioso1a, Isaías Arriola-Quiroz ${ }^{2 b}$, María Cruz-Encarnación ${ }^{2 b}$.}

${ }^{1}$ Facultad de Medicina y Facultad de Salud Pública y Administración, Universidad Peruana Cayetano Heredia. Lima, Perú. Department of Medical Education and Biomedical Informatics, School of Medicine, University of Washington, Seattle, USA. ${ }^{2}$ Sociedad Científica de Estudiantes de Medicina (SOCEMCH), Facultad de Medicina "Alberto Hurtado", Universidad Peruana Cayetano Heredia. Lima, Perú.

aMédico especialista en Informática Biomédica. Maestro en Salud Pública.

${ }^{b}$ Estudiante de Medicina

\section{REFERENCIAS}

1. SciELO. Scientific Electronic Library Online. http: //www.scielo.org/index.php?lang=en. Accesado el 3 de marzo de 2008.

2. Reyes H. La Revista Médica de Chile en una biblioteca de ciencias en la Internet. Rev Med Chile 2001; 129: 131-2. http: //www.scielo.cl/scielo.php?pid=S0034-98872001000200001\&script=sci arttext. Accesado el 3 de marzo de 2008.

3. Huth EJ. The Information explosion. Bull New York Acad Sciences 1989; 65: 647-61.

4. Horna P, Curioso W, Guilés C, Torres C, Kawano J. Conocimientos, habilidades y características del acceso a Internet en estudiantes de medicina de una Universidad Peruana. Anales de la Facultad de Medicina 2002; 63: 32-9.

5. Curioso WH, Montori V, Curioso WI. Medicina basada en evidencias para el gastroenterólogo. Rev Gastroenterol Peru 2004; 24: 75-91. http: //www.scielo.org.pe/scielo.php?script=sci_arttext\&pid=S1022-51292004000100008\&Ing=es\&nrm=iso\&tIng=es. Accesado el 3 de marzo de 2008. 